\title{
Controle on-line da taxa média de defeitos por item produzido numa produção finita
}

\author{
Carla Simone de Lima Teixeira ${ }^{\text {a* }}$, Pledson Guedes de Medeiros ${ }^{\mathrm{b}}$, Linda Lee $\mathrm{Ho}^{\mathrm{c}}$ \\ ${ }^{a *}$ carla.cslt@gmail.com, IFRN, Brasil \\ bpledson@ccet.ufrn.br, UFRN, Brasil \\ clindalee@usp.br, USP, Brasil
}

\begin{abstract}
Resumo
Este trabalho propõe uma abordagem para monitoramento da taxa média de defeitos por item produzido numa produção finita ou encomenda de $\mathrm{N}$ itens. A cada ciclo de $\mathrm{m}$ itens produzidos, inspecionam-se os últimos $\mathrm{r}$ itens. Em cada item inspecionado conta-se o número de defeitos e cada item é classificado como aprovado se o número de defeitos satisfizer o critério do limite de controle. Se todos os $r$ itens forem aprovados, a produção continua, caso contrário interrompe-se a produção à procura de causas especiais. Os itens inspecionados são descartados somente quando há parada no processo. Após a produção de $\mathrm{N}$ itens, um lote adicional será produzido para completar a quantia encomendada, mas esses não passarão por inspeção. Será utilizada uma cadeia de Markov finita de estados discretos para determinar as probabilidades de mudança de estado. Elas são utilizadas nas expressões de custo para determinar a estratégia ótima de monitoração, que será obtida através da otimização de três parâmetros: intervalo amostral $(\mathrm{m})$, tamanho da amostra retrospectiva (r) e o limite de controle (LC). Os parâmetros serão obtidos através de busca direta, de forma que se minimize a expressão do custo médio por item produzido. Um exemplo numérico ilustra a proposta.
\end{abstract}

\section{Palavras-chave}

Controle on-line. Número de não conformidades por item produzido. Produção finita. Modelo econômico.

\section{Introdução}

No atual mercado competitivo, a qualidade tem se mostrado um dos principais conceitos qualificadores das empresas ante a concorrência. Principalmente após os anos 1980, quando a revolução da qualidade exigiu que as empresas investissem na melhoria de seus produtos e processos para sobreviverem no mercado.

Segundo Taguchi (1985), o emprego de processos de inspeção e de ajuste e o uso de sistemas de controle automático são alguns dos métodos que constituem o chamado controle de qualidade on-line. Para Srivastava e $\mathrm{Wu}$ (1991), a proposta de controle de qualidade on-line é produzir produtos uniformes ajustando o processo de acordo com informações obtidas do próprio processo, analisando cada característica de qualidade ou fatores do processo que afetam o produto.

Taguchi, Elsayed e Hsiang (1989) formularam um dos procedimentos de controle baseado em abordagem econômica on-line mais difundidos pela sua simplicidade e facilidade de implantação. A proposta consiste em retirar um único item da linha de produção a cada intervalo fixo de $m$ itens produzidos. Se o valor da característica de interesse do item inspecionado ultrapassar os limites de controle, interrompe-se o processo para investigação e ajuste. Taguchi, Elsayed e Hsiang (1989) argumentam que esse sistema de controle de qualidade on-line deve ser empregado de modo que os valores-alvo desejados da característica de qualidade possam ser economicamente controlados. 0 problema se resume em determinar o intervalo ótimo $(\mathrm{m})$ e o intervalo entre os limites de controle ( $2 \mathrm{~d}$ ) tais que minimizem o custo médio do sistema de controle.

Entre outros trabalhos propostos sobre controle on-line de processos estão os de Adams e Woodall (1989), Nayebpour e Woodall (1993), Borges, Ho e Turnes (2001), Trindade, Ho e Quinino (2007a, b). 
Rodrigues (2009) propõe um modelo de monitoramento on-line para o número médio de defeitos por item através da otimização do intervalo amostral e dos limites de controle que minimizam o custo por item produzido. Silva (2010) também propõe um modelo para monitoramento do número médio de defeitos por item, porém considera a possibilidade de executar ajustes preventivos (ou seja, não ocorre inspeção). É importante ressaltar que essas abordagens consideram processos contínuos (intermitentes), porém, em alguns casos, a produção é realizada a partir de lotes com tamanhos definidos.

Ho e Trindade (2009) propõem um modelo de controle on-line da qualidade para variáveis em uma produção com horizonte finito que visa minimizar o custo médio esperado por item produzido, por meio da determinação da estratégia ótima, otimizando os seguintes parâmetros: intervalo entre as inspeções, limites de controle e tamanho da amostra (a ser inspecionada através de inspeção retrospectiva). A importância dessa abordagem reside no fato de os modelos anteriores não serem aplicáveis a horizontes finitos de produção.

Porém, em muitos casos a qualidade do produto não é atestada através de mensuração. Assim, neste trabalho será proposta uma abordagem motivada pelo modelo apresentado por Ho e Trindade (2009), considerando agora um processo cujo monitoramento é feito por atributos, através da análise do número de não conformidades em uma amostra de itens.

Portanto, o trabalho contribuirá por tratar de um contexto ainda pouco explorado: controle on-line no monitoramento da taxa média de não conformidades por item produzido em uma produção de horizonte finito. Além disso, tem amplo potencial de uso prático em diversos processos produtivos que poderiam se encaixar no modelo proposto, tais como processos de produção de tecido em lotes ou processos de confecção (de roupas), para os quais o produto final é fortemente influenciado pela moda, ou produção do tipo job shop.

Finalmente, o objetivo deste trabalho é desenvolver um modelo probabilístico para monitoramento on-line da taxa média de defeitos por item produzido em processos produtivos de horizonte finito utilizando inspeção retrospectiva. A estratégia ótima será obtida através da otimização dos seguintes parâmetros: o intervalo amostral $(\mathrm{m})$, o tamanho da amostra a ser inspecionada retrospectivamente $(r)$ e o limite de controle (LC). Esses parâmetros foram obtidos através de busca direta, com o fim de minimizar o custo médio por item produzido.

0 artigo está estruturado da seguinte forma: a seção 2 apresenta o modelo probabilístico proposto. 0 custo médio do sistema de controle está apresentado na seção 3. Na seção 4, um exemplo numérico ilustra o efeito da variação dos parâmetros valores de $m, r$ e LC sobre o custo médio do sistema de controle. As conclusões e sugestões são apresentadas na seção 5.

\section{Modelo probabilístico}

Considere que itens são produzidos um a um. 0 processo está em controle estatístico (estado 1) casos eles tenham sido produzidos segundo uma taxa média de defeitos por item $\lambda_{0}$. Num tempo aleatório, a taxa média de defeitos por item aumenta para $\lambda$, assim, diz-se que o processo saiu de controle (estado 11). A probabilidade de mudança do estado 1 para o estado 11 , a cada item produzido, é dada por uma distribuição geométrica de parâmetro $\pi$, sendo $0<\pi<1$. 0 sistema de controle consiste em inspecionar os $r \geq 2$ últimos itens após a produção de $\mathrm{m}$ itens. Isso ocorre até a produção do $n$-ésimo item do lote. Um lote adicional (que não sofrerá inspeção), será produzido e entregue ao cliente, após a produção de $\mathrm{N}$ itens, para que ele não tenha prejuízos quanto à quantidade de produtos encomendada ao final de todos os ciclos. Neste artigo, a estatística monitorada é o número de defeitos no item produzido (D). Um item inspecionado é considerado aprovado caso $\mathrm{D} \leq \mathrm{LC}$ onde LC é o limite superior de controle (o limite inferior será considerado zero). Se todos os $r$ itens forem considerados aprovados, o processo continua operando; caso contrário, o processo é parado para ajuste (critério de ajuste) e reinicia sob o estado 1. Entre o instante em que os itens são inspecionados e o instante em que o processo é parado não são produzidos itens. 0 processo produzido sob o estado 11 só pode retornar ao estado 1 após um ajuste do processo. Caso ocorra uma parada para ajuste do processo os $r$ itens inspecionados são descartados.

0 processo de inspeção pode ser modelado como uma cadeia de Markov finita considerando o conjunto de estados discretos $E$, definidos por um par de índices $(u, w): E=\{00,01,10,11,20,21,30,31\}$, onde 0 indice $u$ indica o real estado do processo em que os itens que compõem o ciclo foram produzidos. Quando $\mathrm{u}=0$, todos os itens foram produzidos no estado 1; se $u=1$, ocorreu uma mudança do estado 1 para o estado 11 no atual ciclo de inspeção e apenas itens produzidos no estado 11 foram incluídos entre os $r$ itens inspecionados; se $\mathrm{u}=2$, também ocorreu uma mudança de estado no ciclo de inspeção, porém pelo menos um item produzido no estado 1 está incluído entre os $r$ inspecionados; e se $u=3$, todos os itens do ciclo foram produzidos no estado ll. 0 índice $w$, por sua vez, assume dois valores: $\mathrm{w}=0$ indica que todos os itens inspecionados foram considerados aprovados; e $\mathrm{w}=1$ se pelos menos um dentre os $\mathrm{r}$ não foi aprovado. A Figura 1 apresenta o fluxograma do processo de monitoramento. 


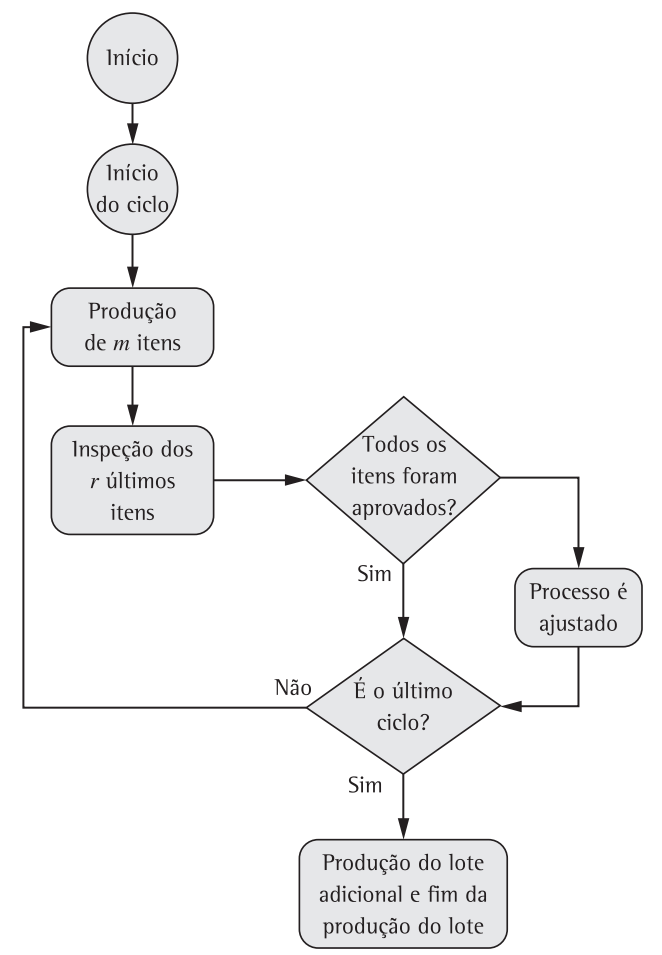

Figura 1. Fluxograma do processo de monitoração.

Para exemplificar a notação utilizada, $\mathrm{P}_{(00)(01)}$ representa a probabilidade de que a inspeção $i$ tenha ocorrido no estado 00 e que a inspeção atual $(i+1)$ ocorra sob o estado 01 , ou simplesmente $P_{(00)(01)}=P\left(E_{i+1}=01 \mid E_{i}=00\right)$. Ou seja, na inspeção anterior as $m$ peças foram produzidas no estado 1 e todos os $r$ itens inspecionados foram aprovados, consequentemente, o processo continua operando normalmente, sem intervenção; e na inspeção atual as $m$ peças também foram produzidas no estado l, porém, pelo menos um dentre os $r$ itens inspecionados não foi considerado aprovado e, consequentemente, o processo é paralisado para ajuste. As probabilidades de transição estão detalhadas a seguir.

A probabilidade $\mathrm{P}_{(00)(00)}$ indica que todos os itens foram produzidos no estado 1 e os $r$ itens inspecionados foram aprovados. Sua probabilidade é dada por

$$
\begin{aligned}
& P_{(00)(00)}=P\left(\Theta_{m}=0\right) \cdot \prod_{i=1}^{r} P\left(D_{i} \leq L C \mid \lambda=\lambda_{0}\right) \\
& =(1-\pi)^{m} \cdot(1-\alpha)^{r}
\end{aligned}
$$

onde

$$
\operatorname{Pr}\left\{\Theta_{m}=0\right\}=(1-\pi)^{m}
$$

é a probabilidade dos $m$ itens serem produzidos no estado 1; LC é o limite superior de controle; D, o número de defeitos no item inspecionado; e 1- $\alpha$ é a probabilidade de um item inspecionado ser considerado aprovado dado que o processo estava sob controle, dada por

$$
1-\alpha=P\left(D \leq L C \mid \lambda=\lambda_{0}\right)=\sum_{d=0}^{L C} \frac{e^{-\lambda_{0}} \lambda_{0}^{d}}{d !}
$$

Em $\mathrm{P}_{(00)(01)}$ todos os itens do ciclo também foram produzidos no estado 1, porém pelo menos um item inspecionado não foi considerado aprovado, consequentemente o processo foi parado para ajuste. Assim:

$$
\begin{aligned}
& P_{(00)(01)}=P\left(\Theta_{m}=0\right) \cdot\left[1-\prod_{i=1}^{r} P\left(D_{i} \leq L C \mid \lambda=\lambda_{0}\right)\right] \\
& =(1-\pi)^{m} \cdot\left[1-(1-\alpha)^{r}\right]
\end{aligned}
$$

As probabilidades $\mathrm{P}_{(00)(10)}$ e $\mathrm{P}_{(00)(11)}$ indicam que ocorreu uma mudança do estado 1 para o estado 11 no atual ciclo de inspeção e apenas itens produzidos no estado 11 foram incluídos entre os $r$ itens inspecionados, $(u=1) . \mathrm{P}_{(00)(10)}$ indica que a produção foi continuada erroneamente, visto que todos os $r$ itens inspecionados foram considerados aprovados. Já $\mathrm{P}_{(00)(11)}$ indica que pelo menos um entre os itens inspecionados não foi aprovado e o processo foi corretamente paralisado para ajuste $(w=1)$.

$$
\begin{aligned}
& P_{(00)(10)}=\left[1-(1-\pi)^{(m-r)}\right] \cdot \beta^{r} \\
& P_{(00)(11)}=\left[1-(1-\pi)^{m-r}\right] \cdot\left(1-\beta^{r}\right)
\end{aligned}
$$

onde

$\beta=\left(D \leq L C \mid \lambda=\lambda_{1}\right)=\sum_{d=0}^{L C} \frac{e^{-\lambda_{1}} \lambda_{1}^{d}}{d !}$

é a probabilidade do item inspecionado ser considerado aprovado dado que o processo estava fora de controle.

As probabilidades $\mathrm{P}_{(00)(20)}$ e $\mathrm{P}_{(00)(21)}$ também indicam que ocorreu uma mudança do estado 1 para o estado 11 no atual ciclo de inspeção. Porém, diferentemente das probabilidades (5) e (6), pelo menos um item produzido no estado 1 está entre os inspecionados $(u=2) . P_{(00)(20)}$ indica que todos os itens inspecionados foram considerados aprovados e o processo foi erroneamente continuado $(w=0)$. Já a segunda probabilidade indica a parada correta do processo para ajuste, visto que pelo menos um item inspecionado não foi considerado aprovado. 


$$
\begin{aligned}
& P_{(00)(20)}=\left[(1-\pi)^{(m-r)}-(1-\pi)^{m}\right] \cdot \sum_{i=1}^{r-1}(1-\alpha)^{i} \beta^{(r-i)} \\
& P_{(00)(21)}=\left[(1-\pi)^{(m-r)}-(1-\pi)^{m}\right] . \\
& {\left[1-\sum_{i=1}^{r-1}(1-\alpha)^{i} \beta^{(r-i)}\right]}
\end{aligned}
$$

Para as probabilidades restantes, $P_{(10)(30)}, P_{(20)(30)}$, $P_{(30)(30)}, P_{(10)(31)}, P_{(20)(31)}$ e $P_{(30)(31)}$, são consideradas apenas a probabilidade de julgar o processo em controle ou fora de controle, visto que o ciclo já se inicia no estado 11. Assim:

$$
\begin{aligned}
& P_{(10)(30)}=\beta^{r}=P_{(20)(30)}=P_{(30)(30)} \\
& P_{(10)(31)}=\left(1-\beta^{r}\right)=P_{(20)(31)}=P_{(30)(31)}
\end{aligned}
$$

As probabilidades $P_{(00)(30)}=P_{(00)(31)}=0$. Em todos os estados nos quais há ajuste do processo, um novo ciclo de inspeções é iniciado necessariamente sob o estado 1. Portanto, todas as linhas pares da matriz $\mathrm{P}_{(\mathrm{m})}$ são iguais à primeira linha da matriz $\mathrm{P}_{(\mathrm{m})}$. Como o processo só retorna ao estado de produção 1 após um ajuste, as seguintes probabilidades são nulas:

$\mathrm{P}_{(10)(00)}=\mathrm{P}_{(10)(01)}=\mathrm{P}_{(10)(10)}=\mathrm{P}_{(10)(11)}=\mathrm{P}_{(10)(20)=} \mathrm{P}_{(10)(21)}=0$.

Como também

$\mathrm{P}_{(20)(00)}=\mathrm{P}_{(20)(01)}=\mathrm{P}_{(20)(10)}=\mathrm{P}_{(20)(11)}=\mathrm{P}_{(20)(20)=} \mathrm{P}_{(20)(21)}=0$.

Por fim,

$\mathrm{P}_{(30)(00)}=\mathrm{P}_{(30)(01)}=\mathrm{P}_{(30)(10)}=\mathrm{P}_{(30)(11)}=\mathrm{P}_{(30)(20)=} \mathrm{P}_{(30)(21)}=0$.

Inserindo (4)-(11) na matriz $\mathrm{P}_{(\mathrm{m})}$, ela pode então ser reescrita como:

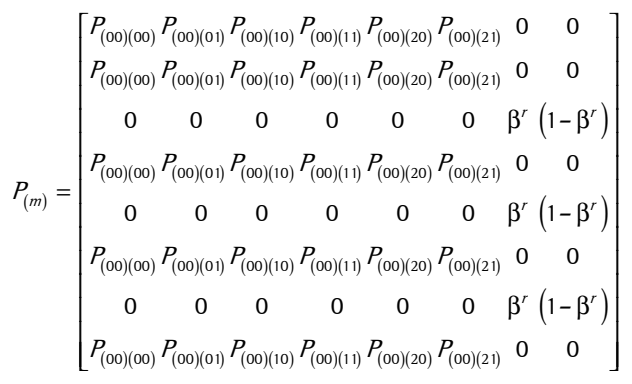

\section{Custo médio do sistema de controle}

Estudos anteriores (TAGUCHI; ELSAYED; HSIANG, 1989; ADAMS; WOODALL, 1989; HO; MEDEIROS; BORGES, 2007) consideraram a existência de três principais custos: de produção de um item nãoconforme, de inspeção e de ajuste. Para o presente estudo, os seguintes custos foram considerados:
- $c_{\mathrm{i}}$ - custo de inspecionar um item;

- $c_{a}$ - custo de ajuste quando a parada ocorrer corretamente (quando de fato, com o processo operando no estado 11);

- $c_{a f}$ - custo de parar o processo quando este estiver operando no estado 1 (alarme falso);

- $c_{n c}$ - custo de enviar um item não conforme (não inspecionado) para etapas posteriores ou para o cliente. Um item é considerado não conforme caso não atenda o limite superior de especificação (LE) (o limite inferior é considerado igual a zero).

- $c_{d n c}$ - custo de descartar um item não conforme;

- $c_{d c}$ - custo de descartar um item conforme entre os $r$ inspecionados quando o processo é paralisado para ajuste.

0 custo de cada estado $(u, w)$ pode ser escrito como:

$c_{(u, w)}=\left(r . c_{i}\right)+\eta_{(u, w)}+\gamma_{(u, w)}+\xi_{(u, w)}$,

em que:

- (r.c.) - custo de inspecionar os $r$ itens a cada ciclo de inspeção (esse custo está incluído em todos os estados da cadeia);

- $\eta_{(\mathrm{u}, \mathrm{w})}$ - custos de envio de itens não conformes, entre os não inspecionados, a estágios subsequentes/ clientes;

- $\gamma_{(\mathrm{u}, \mathrm{w})}$ - custo relacionado ao descarte dos itens inspecionados;

- $\xi_{(u, w)}$ - custo relacionado à parada do processo para ajuste.

Os custos para cada estado da cadeia de Markov estão detalhados a seguir.

\section{- Estado 00}

No estado (00), todos os $m$ itens do ciclo foram produzidos no estado 1 . Dos itens do ciclo, $(m-r)$ não sofrem inspeção. Assim, $(\mathrm{m}-\mathrm{r}) \delta_{1}$ é o número esperado de itens não conformes. Portanto:

$\eta_{(0,0)}=c_{n c}(m-r) \delta_{1}$

onde $\delta_{1}$ é a probabilidade do item ser declarado não conforme quando o processo está sob controle, isto é, $\delta_{1}=\mathrm{P}\left(\mathrm{D}>\mathrm{LE} \mid \lambda=\lambda_{0}\right)$.

Não houve parada no processo $(w=0)$, portanto, nenhum item foi descartado e os $m$ itens do ciclo são enviados. Assim: $\gamma_{(0,0)}=0$. Como não há ajuste no processo então $\xi_{(0,0)}=0$.

\section{- Estado 01}

No estado (01), todos os $m$ itens do ciclo também são produzidos no estado $1, \log 0 \eta_{(0,1)}=\eta_{(0,0)}$. Porém, dentre os $r$ itens inspecionados, pelo menos um deles não foi aprovado. Dessa forma, ocorreu uma parada para o ajuste do processo e os $r$ itens foram descartados. Assim, o custo de descarte é: 


$$
\gamma_{(0,1)}=\sum_{i=1}^{r}\left[(r-i) c_{d c}\left(1-\delta_{1}\right)+i c_{d n c} \delta_{1}\right]
$$

Nesse estado, a parada do processo se dá erroneamente. Assim: $\xi_{(0,1)}=c_{a f}$

\section{- Estado 10}

No estado (10) ocorreu mudança do estado 1 para o estado 11 no atual ciclo. Sabe-se que quando $u=1$, apenas itens produzidos no estado 11 são incluídos entre os inspecionados. Portanto, há a probabilidade de, entre os não inspecionados, haver itens produzidos no estado 11. Assim:

$$
\eta_{(1,0)}=c_{n c} \sum_{i=1}^{m-r} \frac{(1-\pi)^{i-i} \pi}{1-(1-\pi)^{m}}\left[i \delta_{1}+(m-r-i) \delta_{2}\right]
$$

onde $\delta_{2}$ é a probabilidade de o item ser declarado não conforme quando o processo está fora de controle, isto é, $\delta_{2}=\mathrm{P}\left(\mathrm{D}>\mathrm{LE} \mid \lambda=\lambda_{1}\right)$.

Nesse estado, nenhum item foi descartado, pois não houve parada no processo, assim: $\gamma_{(1,0)}=0$. Nesse caso não houve ajuste do processo, então $\xi_{(1,0)}=0$.

\section{- Estado 11}

No estado (11), $\eta_{(1,1)}=\eta_{(1,0)}$. Sabe-se que quando $w=1$, pelo menos um dentre os itens inspecionados não foi aprovado, assim os $r$ itens são descartados. Quando $u=1$, os $r$ itens foram produzidos no estado 11. Portanto:

$$
\gamma_{(1,1)}=\sum_{i=1}^{r}\left[(r-1) c_{d c} \delta_{2}+i c_{d n c}\left(1-\delta_{2}\right)\right]
$$

0 processo é ajustado quando opera no estado 11. Assim: $\xi_{(1,1)}=c_{a}$.

\section{- Estado 20}

No estado (20) também ocorreu mudança de estado no ciclo, todos os itens inspecionados foram considerados aprovados, porém pelo menos um dentre os $r$ itens inspecionados foi produzido no estado 11. Assim: $\eta_{(2,0)}=\eta_{(0,0)}$. Não houve descarte e nem ajuste, então $\gamma_{(2,0)}=0$ e $\xi_{(2,0)}=0$.

\section{- Estado 21}

0 custo de enviar itens não-conformes (entre os não inspecionados) no estado (21) será igual ao estado $(2,0)$, ou seja, $\eta_{(2,1)}=\eta_{(2,0)}$. Sabe-se que quando $u=2$, pelo menos um dentre os $r$ itens foi produzido no estado 1 e $(\mathrm{w}=1)$ indica que pelo menos um dentre os r itens inspecionados foi considerado não aprovado, implicando no descarte dos itens inspecionados. Assim:

$$
\begin{aligned}
& \gamma_{(2,1)}=\sum_{i=m-r-1}^{m} \frac{(1-\pi)^{i-1} \pi}{(1-\pi)^{(m-r)}-(1-\pi)^{m}}(i-m+r) \\
& {\left[c_{d n c} \delta_{1}+c_{d c}\left(1-\delta_{1}\right)\right]+(m-i)} \\
& {\left[c_{d n c} \delta_{2}+c_{d c}\left(1-\delta_{2}\right)\right]}
\end{aligned}
$$

E, com a parada para ajuste do processo (que estava no estado 11), $\xi_{(2,1)}=c_{a}$.

\section{- Estado 30}

No estado (30), o ciclo já se inicia no estado 11, assim:

$\eta_{(3,0)}=c_{n c}(m-r) \delta_{2}$

Como não há itens descartados nem ajuste: $\gamma_{(3,0)}=0$ e $\xi_{(3,0)}=0$.

\section{- Estado 31}

No estado (31), o custo de envio de itens não conformes entre os não inspecionados é igual a todo estado (30), ou seja $\eta_{(3,1)}=\eta_{(3,0)}$. Os $r$ itens que serão descartados foram produzidos no estado 11 , assim: $\gamma_{(3,1)}=\gamma_{(1,1)}$. 0 processo é ajustado no estado 11, assim: $\xi_{(3,1)}=c_{a}$.

Como descrito anteriormente, ao final de $n=\left[\frac{\mathrm{N}}{\mathrm{m}}\right]$ ciclos será produzido um lote adicional para repor, em média, os itens descartados. 0 número esperado de itens descartados será calculado a partir do complementar do número esperado de ciclos onde todos os itens inspecionados são declarados aprovados, ou seja, quando $w=0$. Uma maneira de obter a probabilidade de declarar os $r$ itens inspecionados como aprovados $\left(\mathrm{P}_{\text {aprov }}\right)$ seria utilizar as probabilidades correspondentes na distribuição estacionária da cadeia de Markov, porém essa probabilidade não pode ser obtida desse modo no caso de produções de horizonte finito.

Uma aproximação razoável para isso é considerar a probabilidade de se estar no estado (j) após $\left[\frac{N}{m}\right]$ ciclos dada por

$\pi_{(j)} \approx \frac{1^{\prime} P_{(i, j)}^{n}}{1^{\prime} 1}, i \in E ;$

onde $\mathrm{E}=\{(00),(01),(10),(11),(20),(21),(30),(31)\}$, $P_{(i, j)}^{n}$ é a matriz de transição após $\mathrm{n}$ ciclos; 1 denota um vetor onde todos os elementos são iguais a um. A probabilidade de declarar os $r$ itens inspecionados como aprovados $\left(\mathrm{P}_{\text {aprov }}\right)$ é dada por:

$\mathrm{P}_{\text {aprov }}=\pi_{(00)}+\pi_{(10)}+\pi_{(20)}+\pi_{(30)}$

onde $\pi_{(00)}, \pi_{(10)}, \pi_{(20)}$ e $\pi_{(30)}$ denotam a aproximação da probabilidade estacionária dos estados (00), (10), (20) e (30), respectivamente (todos os $r$ itens inspecionados são declarados aprovados), depois de $\left[\frac{N}{m}\right]$ ciclos, independente do estado inicial. Dessa 
forma, pode-se dizer que o número esperado de ciclos onde todos os itens são declarados aprovados $\left(\mathrm{N}_{\text {aprov }}\right)$ é dada por:

$$
N_{\text {aprov }}=P_{\text {aprov }}\left[\frac{N}{m}\right]
$$

E, consequentemente, o número esperado de itens descartados, que irão compor o lote adicional $\left(\mathrm{E}_{\text {mad }}\right)$, é expresso por:

$$
E\left(m_{a d}\right)=\left[\left[\frac{N}{m}\right]-N_{\text {aprov }}\right] r
$$

Esse lote adicional não sofrerá inspeção e descarte. Baseando-se em Bessegato (2009), tem-se que o custo relacionado a esse lote se reduzirá ao custo de envio de itens não conformes ao mercado. Seja $\mathrm{c}_{\mathrm{ad}}$ o custo médio do lote adicional, se o processo visita todos os estados possíveis, no último ciclo de monitoramento pode-se dizer que a produção do lote adicional iniciará operando no estado 1 . A quantidade esperada de itens não conformes enviada ao mercado é dada por:

$$
\begin{aligned}
& E\left(i_{n c}\right)=\left[m_{a d} \alpha(1-\pi)^{m_{a d}}\right]+\sum_{j=1}^{m_{a d}}(1-\pi)^{j-1} \pi \\
& {\left[(j-1) \alpha+\left(m_{a d}-j+1\right)(1-\beta)\right]}
\end{aligned}
$$

A primeira parcela da expressão (24) se refere à probabilidade de enviar itens não conformes ainda que o processo não mude de estado na produção do lote adicional. Na segunda parcela da expressão é considerada uma mudança de estado na produção do lote. Assim, o custo relacionado ao envio de itens não conformes do lote adicional ao mercado é dado por:

$c_{a d}=c_{n c} E\left(i_{n c}\right)$

Ho e Trindade (2009) definem que o custo médio por produto manufaturado deve ser calculado como uma soma dos custos de todos os ciclos de inspeção realizados. Inicialmente será definida a distribuição inicial do processo. Por definição, o processo produtivo se inicia no estado 1 e o vetor probabilidade que representa a distribuição inicial é dada por:

$\Pi=\left[\begin{array}{llllllll}1 & 0 & 0 & 0 & 0 & 0 & 0 & 0\end{array}\right]$

Assim, utilizando-se as propriedades da cadeia de Markov, a probabilidade de que a cadeia esteja no $i$-ésimo estado depois de $n$ ciclos de inspeções é a $i$-ésima entrada do vetor:

$\Pi^{(\mathrm{n})}=\Pi\left(\mathrm{P}_{(\mathrm{m})}\right)^{\mathrm{n}}$
Consequentemente, o custo relacionado com o $n$-ésimo ciclo de inspeção é dado por $\Pi\left(\mathrm{P}_{(\mathrm{m})}\right)^{\mathrm{n}} \mathrm{C}_{(\mathrm{m})}$, onde $\mathrm{C}_{(\mathrm{m})}$ é o vetor de custos, definido como:

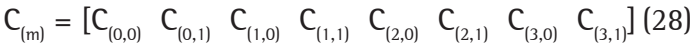

em que cada elemento é o custo de cada estado (uw), definidos em (13). Note-se que este vetor é função do tamanho do intervalo amostral $m$, razão da existência do índice $(\mathrm{m})$.

A expressão do custo médio por item produzido é feita pela seguinte expressão:

$$
C=\frac{1}{N^{*}}\left[\sum_{i=1}^{[N / m]} \Pi\left(P_{(m)}\right)^{i} C_{(m)}\right.
$$

$\left.+\left(1-I_{0}\left(m^{*}\right)\right)\left[\Pi\left(P_{(m)}\right)^{[N / m]} P_{\left(m^{*}\right)} C_{\left(m^{*}\right)}\right]\right]$

$+\frac{1}{m_{a d}} c_{a d}$

onde $N^{*}$ representa $\left(N-m_{a d}\right) ; m^{*}$ representa o último ciclo, no caso de o intervalo entre as inspeções não ser múltiplo do tamanho do lote, e $l_{o}$ representa uma função indicadora que assume valor 1 quando o argumento é verdadeiro.

0 problema consiste em determinar $m, r$ e $L C$ que minimizam (30), ou seja:

$$
\left(m^{\circ}, r^{\circ}, L C^{\circ}\right)=\arg \underbrace{\min }_{(m, r, L C)} C
$$

\section{Exemplo numérico}

0 modelo proposto será ilustrado por um exemplo numérico. Considere uma fábrica de biquinis que quer instalar um sistema de controle do número médio de defeitos por peça. 0 setor de confecção de biquinis apresenta características próprias, por exemplo, ser fortemente influenciado pela moda e pela exigência de exclusividade por parte dos consumidores. 0 volume de produção de cada modelo deve ser pequeno, para garantir a satisfação dos consumidores.

Considere que o processo de manufatura de biquinis tem os seguintes custos:

- custo de inspeção $c_{i}=\$ 0,6$;

- custo de envio de um item não conforme $c_{n c}=\$ 6,0$;

- custo de ajuste $c_{a}=\$ 60$;

- custo de descartar um item conforme $c_{d c}=\$ 2,0$;

- custo de descartar um item não conforme $c_{\mathrm{dnc}}=\$ 1,0$;

- custo de parar o processo no estado 1 (alarme falso) $c_{f}=\$ 3,0$. 
E os seguintes parâmetros de processo:

- probabilidade de mudança de estado: $\pi=0,001$;

- número médio de defeitos por item no estado 1 : $\lambda_{0}=2$

- número médio de defeitos por item no estado 11: $\lambda_{1}=6,5$;

- limite superior de especificação: $\mathrm{LE}=5$;

- tamanho do lote de produção: $\mathrm{N}=250$.

No modelo de monitoração, o processo é parado caso pelo menos um dentre os $r$ não for considerado aprovado. A Figura 2 mostra o custo médio do sistema de controle por item produzido: (a) em função do intervalo entre as inspeções $(m)$; (b) em função do tamanho da amostra retrospectiva; e (c) em função do limite de controle $(L C)$. Os valores ótimos obtidos são $m^{0}=14, r^{0}=2$ e $L C^{0}=7$, que resultam em um custo médio por item produzido de \$0,2926.

Para fins de comparação, analisou-se também o caso de o fabricante optar por descartar todos os itens inspecionados independentemente do resultado da inspeção. Isso resultou nos valores ótimos: $m^{\circ}=50, r^{\circ}=2, L C^{\circ}=6$ e um custo médio por item $\mathrm{C}=\$ 0,4582$, ou seja, aproximadamente $63 \%$ maior que o obtido quando se descartam os itens apenas quando o processo é parado para ajuste. Caso o fabricante opte pela envio da produção toda (sem fazer inspeção, incidiria apenas o custo de envio de itens não conformes), o custo elevaria (a)

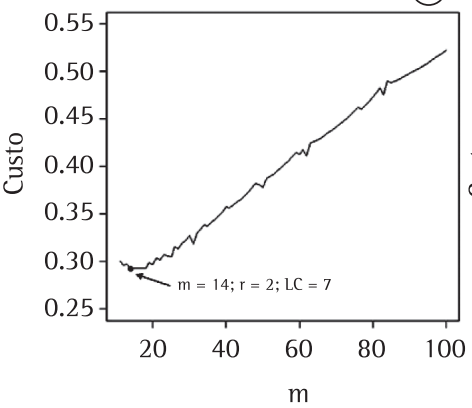

(b)

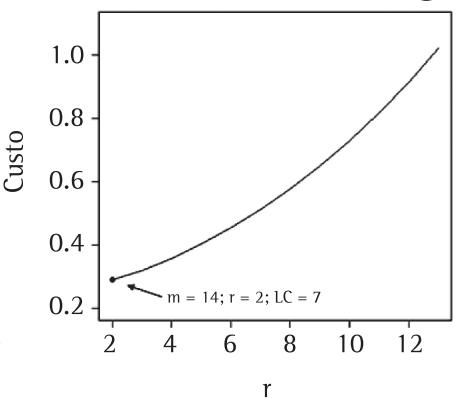

(c)

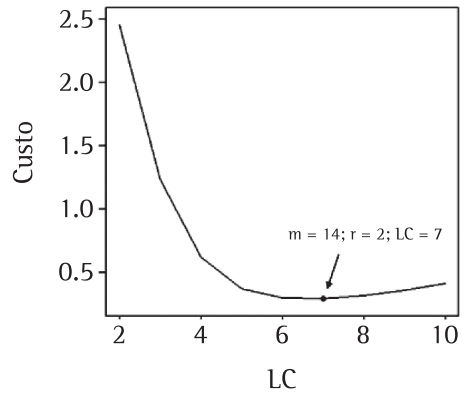

Figura 2. (a) Gráfico de $m \times C$, com $r=2$ e $L C=7$; (b) Gráfico de $r \times C$, com $m=14$ e $L C=7$; (c) Gráfico de $L C \times C$, com $m=14$ e $r=2$.

Tabela 1. Determinação de $\mathrm{m}^{0}, \mathrm{r}^{0}, \mathrm{LC}^{0}$ e C para uma variação individual dos parâmetros do modelo, mantidas as demais constantes nos valores utilizados no exemplo numérico.

\begin{tabular}{|c|c|c|c|c|c|c|c|c|c|}
\hline$\pi$ & $\mathrm{m}$ & $r$ & $\mathrm{LC}$ & C & $\lambda_{1}$ & $\mathrm{~m}$ & $r$ & $\mathrm{LC}$ & C \\
\hline 0,0001 & 62 & 2 & 7 & 0,1736174 & 4 & 31 & 2 & 7 & 0,2794721 \\
\hline 0,0005 & 21 & 2 & 7 & 0,2312762 & 4,5 & 21 & 2 & 6 & 0,2992703 \\
\hline 0,0010 & 14 & 2 & 7 & 0,2926477 & 5 & 18 & 2 & 6 & 0,3013037 \\
\hline 0,0050 & 8 & 2 & 7 & 0,6614244 & 6,5 & 14 & 2 & 7 & 0,2926477 \\
\hline 0,0100 & 6 & 2 & 7 & 1,0725710 & 11 & 8 & 2 & 7 & 0,2661783 \\
\hline LE & $\mathrm{m}$ & $r$ & $\mathrm{LC}$ & C & $c_{i}$ & $\mathrm{~m}$ & $r$ & LC & C \\
\hline 1 & 8 & 7 & 8 & 1,1396150 & 0,06 & 5 & 4 & 8 & 0,1554907 \\
\hline 3 & 4 & 3 & 8 & 0,7398937 & 0,3 & 11 & 2 & 7 & 0,2448334 \\
\hline 5 & 14 & 2 & 7 & 0,2926477 & 0,6 & 14 & 2 & 7 & 0,2926477 \\
\hline 7 & 25 & 2 & 7 & 0,1740943 & 1,2 & 31 & 2 & 6 & 0,3443905 \\
\hline 10 & 62 & 2 & 10 & 0,0941636 & 6 & 62 & 2 & 6 & 0,5482628 \\
\hline$c_{d c}$ & $\mathrm{~m}$ & $r$ & $\mathrm{LC}$ & C & $c_{d n c}$ & $\mathrm{~m}$ & $r$ & $\mathrm{LC}$ & C \\
\hline 1 & 14 & 2 & 7 & 0,2918458 & 0,5 & 14 & 2 & 7 & 0,2920715 \\
\hline 2 & 14 & 2 & 7 & 0,2926477 & 1 & 14 & 2 & 7 & 0,2926477 \\
\hline 4 & 14 & 2 & 7 & 0,2942514 & 2 & 14 & 2 & 7 & 0,2937999 \\
\hline 10 & 14 & 2 & 7 & 0,2990625 & 5 & 14 & 2 & 7 & 0,2972568 \\
\hline 200 & 18 & 2 & 7 & 0,4419560 & 100 & 18 & 2 & 7 & 0,406212 \\
\hline$c_{n c}$ & $\mathrm{~m}$ & $r$ & $\mathrm{LC}$ & C & $c_{f}$ & $\mathrm{~m}$ & $r$ & $\mathrm{LC}$ & C \\
\hline 0,6 & 62 & 2 & 10 & 0,09756284 & 0,3 & 14 & 2 & 7 & 0,2922314 \\
\hline 6 & 14 & 2 & 7 & 0,2926477 & 3 & 14 & 2 & 7 & 0,2926477 \\
\hline 12 & 8 & 2 & 7 & 0,4258789 & 6 & 14 & 2 & 7 & 0,2931102 \\
\hline 30 & 5 & 2 & 7 & 0,7061944 & 30 & 18 & 2 & 7 & 0,2963088 \\
\hline 600 & 10 & 9 & 8 & 3,3337780 & 300 & 14 & 2 & 8 & 0,3280848 \\
\hline
\end{tabular}


para $\mathrm{C}=\$ 0,8060$ (mais que o dobro do obtido pelo modelo proposto).

A Tabela 1 mostra os valores ótimos de $m, r$ e $L C$ variando apenas um parâmetro por vez e mantidos os demais constantes nos valores utilizados no exemplo numérico do início desta seção.

De acordo com a Tabela 1, conforme a probabilidade de mudança de estado aumenta, o custo médio por item produzido também aumenta, pois há mais paradas para inspeção e/ou ajuste. 0 intervalo entre as inspeções, por sua vez, diminui conforme o aumento da probabilidade de mudança, para detectar mais rapidamente a mudança de estado.

Para valores de $\lambda_{1}$ menores ou iguais ao limite de especificação $(\mathrm{LE}=5)$, custo médio por item produzido aumenta e intervalo amostral diminui conforme $\lambda_{1}$ aumenta, pois a tendência é inspecionar peças com maior frequência. Para valores acima do limite de especificação, o custo vai reduzindo conforme $\lambda_{1}$ aumenta e o intervalo amostral (m) tende a aumentar.

Aumentos no custo de inspeção implicam em uma redução da frequência de ocorrência de inspeções e uma redução gradual no limite de controle. No caso do custo de inspeção ser 0,06, o modelo indica uma inspeção praticamente de todos os itens do lote: a cada cinco itens produzidos, inspeciona-se quatro. Além disso, o custo por item também aumenta conforme o custo de inspeção aumenta.

Observa-se que com o aumento do custo de descarte de um item conforme $\left(\mathrm{c}_{\mathrm{dc}}\right)$ o custo médio por item produzido também aumenta, porém numa proporção muito baixa. Para valores do custo de descarte extremos $\left(c_{d c}=200\right)$, o intervalo entre as inspeções aumenta, numa tentativa de se descartar menos itens. 0 tamanho da amostra retrospectiva se mantém constante independentemente do valor de $\mathrm{c}_{\mathrm{dc}}$, explicado pela tentativa de diminuir a quantidade de itens inspecionados e, consequentemente, descartados.

0 mesmo se observa com a variação do custo de descarte de um item não conforme $\left(\mathrm{c}_{\mathrm{dnc}}\right)$, onde o intervalo amostral diminui somente para valores 100 vezes maiores que o valor de $c_{\text {dnc }}$ fixado. Vale salientar que esse custo está presente apenas nos estados onde há parada do processo, ou seja, quando pelo menos um entre os $r$ itens inspecionados não é aprovado.

Para variações no custo de produção de um item não conforme e envio deles para etapas posteriores ou para os clientes $\left(\mathrm{c}_{\mathrm{nc}}\right)$, o valor de $m$ decresce à medida que o custo aumenta, já que nessa situação é necessário identificar e descartar o maior número possível de itens não conformes. No caso mais extremo ( $c_{n c} 100$ vezes maior que o fixado), o modelo indica inspeção de, praticamente, todas as peças do lote $(m=10$ e $r=9)$.

Embora os resultados da Tabela 1 sejam muito interessantes, eles não indicam quais parâmetros de entrada (oito parâmetros no total, sendo cinco de custos e três de processo) causam grande impacto no custo, comparativamente. A expressão (29) (custo médio por item produzido) sabidamente não é linear. No entanto, numa vizinhança pequena em relação aos valores de entrada (descritos no início da seção 4), a expressão (29) pode ser aproximada por uma função linear. Para variar todos os parâmetros simultaneamente, obedeceu-se à estrutura de um planejamento de experimento considerando-se a execução de 16 realizações de experimento fatorial fracionado $2^{(8-4)}$. A escolha do plano recai no critério de menor número de realizações com resolução IV (WU; HAMADA, 2000). 0 nível mais baixo é igual ao especificado no início da seção 4 e o mais alto é igual ao mais baixo $+50 \%$. 0 nome dos fatores (custos e parâmetros do processo), os níveis, os efeitos dos fatores e os respectivos níveis descritivos estão na Tabela 2. Os custos que mais impactam o custo médio por item produzido são o custo de enviar itens não conformes para o cliente $\left(c_{n c}\right)$, ou etapas posteriores, e o custo de inspeção (c); ; e os parâmetros de processo que mais impactam o custo médio por item produzido são a probabilidade de mudança $\pi \mathrm{e}$ o limite de especificação (LE). Na Figura 3 estão os gráficos do intervalo de confiança do custo médio por item produzido em função dos níveis dos fatores. Pode-se observar que as médias dos fatores apontados como impactantes apresentam diferença em função dos níveis dos fatores.

Tabela 2. Efeitos dos fatores e níveis empregados no planejamento de experimento.

\begin{tabular}{|c|c|c|c|c|}
\hline \multirow{2}{*}{ Parâmetros (Fatores) } & \multicolumn{2}{|c|}{ Nivel } & \multicolumn{2}{|c|}{ Custo médio (C) } \\
\hline & Baixo (-) & Alto (+) & Efeito & $\mathrm{p}$-value \\
\hline Probabilidade de mudança de estado: $\pi$ & 0,001 & 0,0015 & 0,058 & 0,002 \\
\hline Número médio de defeitos por item no estado 11: $\lambda_{1}$; & 6,5 & 9,75 & $-0,016$ & ns \\
\hline Limite superior de especificação: LE & 5,0 & 7,5 & $-0,131$ & 0,000 \\
\hline Custo de inspeção $c_{i}$ & 0,6 & 0,9 & 0,035 & 0,024 \\
\hline Custo de envio de um item não conforme $c_{n c}$ & 6,0 & 9,0 & 0,057 & 0,002 \\
\hline Custo de descartar um item conforme $\mathrm{c}_{\mathrm{dc}}$ & 2,0 & 3,0 & 0,001 & ns \\
\hline Custo de descartar um item não conforme $c_{\mathrm{dnc}}$ & 1,0 & 1,5 & 0,002 & ns \\
\hline Custo de parar o processo no estado 1 (alarme falso) $\mathrm{c}_{\mathrm{f}}$ & 3,0 & 4,5 & 0,000 & ns \\
\hline
\end{tabular}


(a)

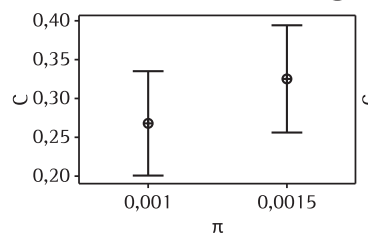

(e)

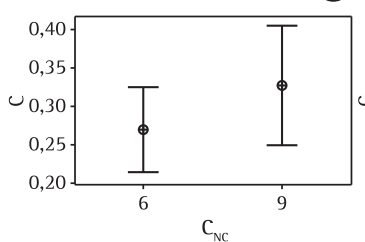

(b)

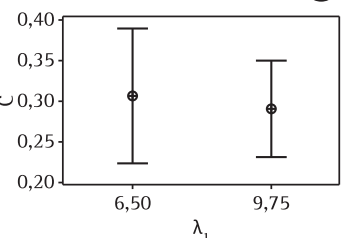

(f)

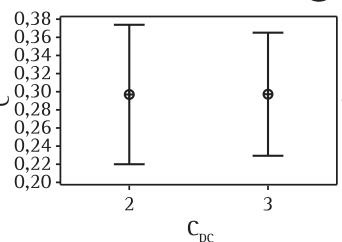

(c)

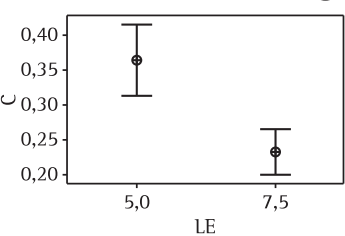

(9)

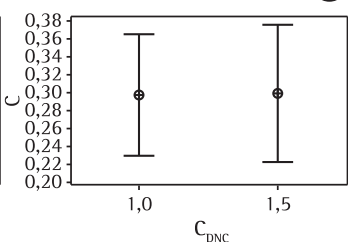

(d)

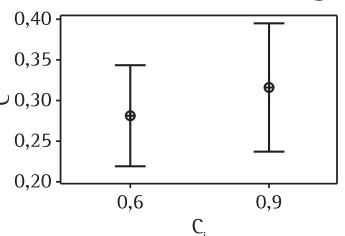

(h)

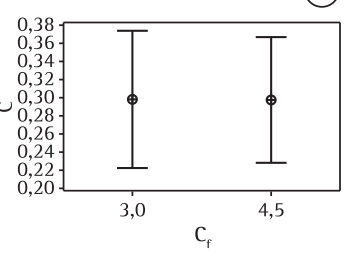

Figura 3. Intervalo de confiança do custo médio por item produzido versus os níveis dos fatores.

Tabela 3. Comportamento do modelo proposto em função do tamanho da encomenda $\mathrm{N}$.

\begin{tabular}{ccccc}
\hline $\mathrm{N}$ & $\mathrm{m}$ & $\mathrm{r}$ & $\mathrm{lC}$ & $\mathrm{C}$ \\
\hline 150 & 15 & 2 & 7 & 0,2969298 \\
250 & 14 & 2 & 7 & 0,2926477 \\
350 & 16 & 2 & 7 & 0,2928142 \\
450 & 16 & 2 & 7 & 0,2917719 \\
550 & 15 & 2 & 7 & 0,2939317 \\
650 & 16 & 2 & 7 & 0,2943243 \\
1000 & 15 & 2 & 7 & 0,2953991 \\
2000 & 16 & 2 & 7 & 0,2978843 \\
5000 & 15 & 2 & 7 & 0,3047272 \\
10000 & 15 & 2 & 7 & 0,3155272 \\
50000 & 12 & 2 & 8 & 0,3816743 \\
100000 & 11 & 2 & 9 & 0,4368249 \\
1000000 & 11 & 2 & 10 & 0,6865796 \\
\hline
\end{tabular}

0 modelo proposto no presente trabalho foi desenvolvido para produções finitas. Na Tabela 3 estão os parâmetros ótimos variando-se o tamanho do lote. Pode-se observar que, para encomendas inferiores a 1.000 itens, o custo médio por item produzido sofre pouca variação e que um intervalo médio de inspeção $m$ igual a 15 unidades e limite de controle igual a 7 poderiam ser empregados, visto que o custo sofreria pouca variação. Porém, para lotes maiores, o modelo resulta em custos muitas vezes mais elevados que os obtidos para os pequenos lotes e há uma variação maior no valor de $m$. Para esses casos, o modelo proposto não funciona adequadamente e, portanto, deve-se adotar o procedimento de monitoramento para produção infinita.

\section{Conclusões e sugestões}

Uma conclusão importante realizada a partir da análise do comportamento dos resultados é: para lotes grandes de produção (valores grandes de N) o modelo proposto mostrou-se ineficaz, resultando num custo médio por item produzido relativamente alto. Os modelos para produções de horizonte infinito devem ser utilizados nesses casos.

Além disso, observa-se que do tamanho da amostra retrospectiva [o valor de (r)] mostrou-se inalterado e sempre igual a dois. Isso pode ser explicado pela tentativa de reduzir o número de itens descartados e, consequentemente, diminuir o custo médio por item produzido.

Para os parâmetros fixados neste trabalho, a estratégia de descartar todos os itens inspecionados ocasiona um aumento no custo médio por item, quando comparada à estratégia de descartar os itens inspecionados apenas quando pelo menos um deles não é aprovado. Além disso, o resultado demonstra que os itens devem ser inspecionados menos frequentemente.

É importante salientar que, na proposição de gráficos de controle para monitoramento da taxa média de não conformidade é usual empregar o número total de não conformidade encontrados na amostra como estatística a ser monitorada visto que sob ponto de vista estatístico, esta estatística, para um fixado valor de risco (probabilidade de parar o processo, quando o mesmo está sob controle), leva a um menor valor de ARL (menor tempo médio para detectar que o processo está fora de controle). Porém, priorizando a abordagem econômica, optou-se por monitorar o número de itens não aprovados numa amostra. Isto numa situação prática evita que todos os $r$ itens sejam, obrigatoriamente, inspecionados. Além disso, monitorar o número total de não conformidades na amostra leva a um modelo diferente do aqui proposto, modificando as expressões utilizadas para obter os parâmetros ótimos.

Para trabalhos futuros, sugere-se o emprego de outras estatísticas de monitoramento como, por 
exemplo, utilizar o número total de defeitos numa amostra de $r$ itens, estender o problema empregando diferentes intervalos de inspeção (um longo e um curto); utilizar mais do que um conjunto de limites (limite de controle e limite de alarme), introduzir outros critérios para decidir o ajuste do processo.

\section{Referências}

ADAMS, B. M.; WOODALL, W. H. An analyses of Taguchi's on-line process-control procedure under a random-walk model. Technometrics, v. 31, n. 4, p. 401-413, 1989. http://dx.doi.org/10.1080/00401706.1989.10488589

BESSEGATO, L. F. Extensão para controle on-line por atributo com erros de classificação: intervalo de inspeção variável, amostragem não-unitária, horizonte finito e infinito. 2009. Tese (Doutorado)-Universidade Federal de Minas Gerais, Belo Horizonte, 2009.

BORGES, W.; HO, L. L.; TURNES, O. An analyses of Taguchi's on-line quality monitoring procedure for attributes with diagnosis errors. Applied Stochastic Models in Business an Industry, v. 17, p. 261-276, 2001. http://dx.doi. org/10.1002/asmb.442

HO, L. L.; MEDEIROS, P. G.; BORGES, W. An alternative model for on-line quality monitoring for variables. International Journal of Production Economics, v. 107, n. 1, p. 202222, 2007. http://dx.doi.org/10.1016/j.jpe.2006.09.002

HO, L. L.; TRINDADE, A. L. G. Economic design of an X chart for short-run production. International Journal of Production Economics, v. 120, p. 613-624, 2009. http:// dx.doi.org/10.1016/j.ijpe.2009.04.012

NAYEBPOUR, M. R.; WOODALL, W. H. An analyses of Taguchi's on-line quality-monitoring procedures for attributes. Technometrics, v. 35, n. 1, p. 53-60, 1993. http://dx.doi.org/10.1080/00401706.1993.10484993

RODRIGUES, R. M. Controle on-line para o número de não-conformidades em um item inspecionado. 2009.
Dissertação (Mestrado)-Universidade Federal do Rio Grande do Norte, Natal, 2009.

SILVA, L. P. Ajuste preventivo versus ajuste corretivo no controle on-line de processo do número de não conformidades num item inspecionado. 2010. Dissertação (Mestrado)-Universidade Federal do Rio Grande do Norte, Natal, 2010.

SRIVASTAVA, M. S.; WU, Y. A second order approximation to Taguchi etal.'s on-line control procedure. Communications in Statistics A, v. 20, p. 2149-2168, 1991. http://dx.doi. org/10.1080/03610929108830625

TAGUCHI, G. Quality Engineering in Japan. Bulletin of the Japan Society of Precision Engineering, 1985.

TAGUCHI, G.; ELSAYED, E. A.; HSIANG, T. Quality Engineering in Production in Systems. New York: McGray-Hill, 1989.

TRINDADE, A. L. G.; HO, L. L.; QUININO, R. C. Controle on-line por atributos com erros de classificação: uma abordagem econômica com classificações repetidas. Pesquisa Operacional, v. 27, p. 105-116, 2007a. http:// dx.doi.org/10.1590/S0101-74382007000100006

TRINDADE, A. L. G.; HO, L. L.; QUININO, R. C. Monitoring process for attributes with quality deterioration and diagnosis errors. Applied Stochastic Models in Business and Industry, v. 23, p. 339-358, 2007b. http://dx.doi. org/10.1002/asmb.675

WU, C. F. J.; HAMADA, M. Experiments - Planning, Analysis, and Parameter Design Optimization. John Wiley \&t sons, Inc., 2000.

\section{Agradecimentos}

Os autores agradecem os revisores anônimos pelas sugestões e recomendações que certamente contribuíram muito para a melhoria do presente artigo. Os autores também agradecem ao CNPq e à CAPES pelo financiamento parcial deste projeto.

\title{
On-line process control of the average number of defects per item in a short run production
}

\begin{abstract}
This paper proposes an approach for monitoring the average number of non-conformities per items in short-run productions of $\mathrm{N}$ items. After every $m$ produced items, the last $r$ items are inspected. For each inspected item, the number of defects is counted; each inspected item is classified as approved if it meets the control limit criterion. If all $r$ inspected items are approved, then the production goes on, otherwise it is stopped for adjustment. The inspected items are all discarded in case of production stoppage. After a production of $\mathrm{N}$ items, an additional lot is produced to complete the size ordered, but this additional lot does suffer inspection. A finite discrete state Markov chain is used to determine the transitory probabilities, which will be considered in terms of cost, to determine the optimal strategy for monitoring, obtained by optimizing three parameters: sampling interval $(\mathrm{m})$, retrospective sample size $(\mathrm{r})$, and control limit (cl). These parameters are obtained computationally by direct search to minimize the expression of the average cost per item produced. A numerical example illustrates the proposal.
\end{abstract}

\section{Keywords}

On-line control. Average number of defects. Short-run production. Economic model. 